\title{
Optimierung der Anwendung von 'Apilife VAR' zur Bekämpfung von Varroa jacobsoni Oud in Bienenvölkern
}

\author{
A Imdorf, V Kilchenmann, C Maquelin, S Bogdanov \\ Sektion Bienen, Forschungsanstalt für Milchwirtschaft, $\mathrm{CH}-3097$ Liebefeld, Schweiz
}

(Eingegangen 19 April 1993; angenommen 14 Juli 1993)

\begin{abstract}
Zusammenfassung - 'Apilife VAR' wurde in Italien zur Bekämpfung der Varroa jacobsoni in Bienenvölkern entwickelt. Es enthält Thymol, Eukalyptol, Menthol und Kampfer und wird im Spätsommer bis Herbst eingesetzt. In der vorliegenden Arbeit werden Untersuchungen betreffend der Behandlungsdauer, Dosierung, Formulierung, Plazierung, Verdunstung und Rückstandsbildung von 'Apilife VAR' dargestellt. Nach einer Behandlungsdauer von 8 Wochen betrug die durchschnittliche Wirksamkeit im Schweizerkasten bei der Dosierung von 2 mal 1 Tafel 97,7\%. Ob 'Apilife VAR' mit oder ohne Kampfer formuliert war, hatte keinen Einfluss auf den Behandlungserfolg. Bei der Behandlung von unten in Magazinbeuten war die Wirksamkeit ungenügend. Es wird eine Methode vorgestellt, um nach der Behandlung mit 'Apilife VAR' die Restmilben im Bienenvolk zu schätzen. In den 2 Wochen nach Behandlungsende entsprach 1 Varroa pro Tag 40 Milben bei der Perizinbehandlung. Im Futter und Honig konnten nur Thymolrückstände nachgewiesen werden. Nach 8 Wochen Behandlung wurden im Oktober im eingelagerten Futter in Schweizerkasten und Magazinbeuten durchschnittliche Rückstandswerte von 2,6 resp 2,0 mg/kg gemessen. Im Frühjahrshonig lagen die entsprechende Werte 8 Monate später bei 0,1 und $0,03 \mathrm{mg} / \mathrm{kg}$.
\end{abstract}

Apis mellifera / Varroa jacobsoni / Bekämpfung / ätherisches Ö / Wirksamkeit / Rückstände

\section{EINLEITUNG}

Auf der Suche nach toxikologisch unbedenklichen Akariziden zur Bekämpfung von Varroa jacobsoni wurden verschiedene ätherische Öle auf ihre varroazide Wirkung getestet (Colin, 1990; Hoppe, 1990; Kraus, 1990). Die meisten dieser Pflanzenextrakte wiesen aber eine ungenügende Wirkung oder eine schlechte Bienenverträglichkeit auf. Einzig eine Extraktmi- schung aus Thymian (Thymus vulgaris) und Salbei (Salvia officinalis) (Colin, 1990) sowie das Wintergrünöl (Hoppe und Ritter, 1989) zeigten bei der Anwendung mit einem Aerosol- oder Warmluftgerät eine Wirkung von 95,4 respektive 93\%.

Eine bessere varroazide Wirkung zeigten Komponenten von ätherischen Ölen wie zum Beispiel Thymol, das von verschiedenen Autoren als Reinsubstanz getestet wurde (Chiesa, 1991 für Literatur- 
übersicht). Je nach Anwendungsart schwankt die Wirksamkeit stark. So erzielten zum Beispiel Marchetti und Barbattini (1984) durch Einhängen von $15 \mathrm{~g}$ Thymolkristallen in einer Gazetasche (4 mal, Intervall 4 Tage) einen durchschnittlichen Behandlungserfolg von $66 \%$. Chiesa (1991), Lodesani et al (1990) und Frilli et al (persönliche Mitteilung) hingegen erreichten mit einer mehrmaligen Behandlung durch Einstreuen von Thymolstaub eine Wirksamkeit von 96,8, 99 und 95\%.

Rickli et al (1991) wendeten das Produkt 'Apilife VAR' an, das Thymol, EucaIyptol, Menthol und Kampfer enthält und erzielte im Schweizerkasten auf zwei Ständen eine durchschnittliche Wirksamkeit von 96,4 respektive $99 \%$. In einzargigen Zanderbeuten erzielten Liebig et al (1992) mit dem gleichen Produkt bei halber Dosierung einen durchschnittichen Behandlungserfolg von $97,4 \%$. Van der Steen (1992) prüfte ebenfalls 'Apilife VAR' sowie zwei Eigenmischungen der gleichen $\mathrm{Zu}$ sammensetzung, einmal mit und einmal ohne Kampfer. Der durchschnittliche Behandlungserfolg lag bei 74,92 und $88 \%$. Erfahrungsberichte von italienischen Imkern (persönliche Mitteilungen) weisen darauf hin, dass im Dadantkasten mit sehr unterschiedlichem Behandlungserfolg zu rechnen ist und dass hin und wieder Brutund Bienenschäden auftreten können. Mautz (1982) berichtete von Bienenschäden, welche nach einer Thymolbehandlung gegen Acarapis woodi aufgetreten waren. In dieser Arbeit wurde die Anwendung von 'Apilife VAR' in verschiedenen Kastentypen, Behandiungsdauer, Dosierung, Formulierung, Plazjerung, Verdunstung und Bienentoxizität untersucht.

\section{MATERIAL UND METHODEN}

Es wurden 9 Bienenstände im schweizerischen Mittelland und Voralpengebiet mit Bienen der
Land - bzw Carnicarasse in die Untersuchung einbezogen. Auf den Ständen 1-7 waren die Völker in Schweizerkästen (Hinterbehandlungsbeute im Warmbau) untergebracht. Die Stände 8 und 9 waren mit Dadant respektive Ritterkästen ausgerüstet (Magazinbeuten im Kaltbau).

Die $5 \times 9 \times 1 \mathrm{~cm}$ grossen Vermikulittafeln waren mit ca $20 \mathrm{~g}$ einer Mischung aus Thymol $(76 \%)$, Eukalypthol $(16,4 \%)$, Menthol $(3,8 \%)$ und Kampfer $(3,8 \%)$ getränkt.

Es wurden folgende Fragen geprüft:

(a) Behandlungsdauer; 4 oder 8 Wochen;

(b) Dosierung im Schweizerkasten: $2 \mathrm{mal} 1 / 2$ Tafel, 2 mal 1 Tafel, oder 1 mal 1 Tafel;

(c) Formulierung: mit oder ohne Kampfer;

(d) Plazierung der Tafel im Schweizerkasten: direkt auf die Brutwaben oder auf ein bienendichtes Gitter über den Brutwaben;

(e) Plazierung der Tafel in Magazinbeuten: direkt auf die Brutwaben (oben) oder unter das Bodengitter des Hochbodens (unten);

Ausser bei (a) betrug die Behandlung jeweils 7-8 Wochen. Die Dosierung war, mit Ausnahme von (b), immer zwei mal 1 Tafel. Die Formulierung war ausser bei (c) immer mit Kampfer.

Während der ganzen Untersuchung wurde wöchentlich der Milbenfall gezählt. Alle Bienenkästen waren mit einer gittergeschützen Unterlage versehen. Vor dem Behandlungsbeginn wurde je nach Stand während 3-6 Wochen der natürliche Milbenfall ausgezählt. Der Milbenfall vom Behandlungsbeginn bis zur ersten Kontrollbehandlung mit Perizin wurde als Behandlungsmilbenfall von 'Apilife VAR' gezählt. Der Wirkungsgrad von Perizin beträgt ca $95 \%$ (Ritter et al, 1986). Auf den Ständen 6 und 7 wurde wegen des geringen Milbenfalls nach der ersten Perizinbehandlung keine zweite durchgeführt. Zum Berechnen des Wirkungsgrades von 'Apilife VAR' wurde der Behandlungsmilbenfall von 'Apilife VAR' und Perizin als die Gesamtmilbenpopulation angenommen. Zur statistischen Auswertung wurde auf jedem Stand zwischen den getesteten Versuchsgruppen die Wahrscheinlichkeit der Nullhypothese mit Hilfe des $t$-Tests ( $P \leq 0,05$ - berechnet).

Die Behandlungen mit 'Apilife VAR' wurden zwischen dem 7.8 und 15.10 .91 und die von Perizin zwischen dem 25.11.91 und 6.1.92 durchgeführt. Durch die relativ grosse Zeitspanne zwischen den beiden Behandlungen wurde eine Verschlechterung des Behandlungserfolges von 
'Apilife VAR' durch mögliche Rückinvasion und Vermehrung der Varroa in Kauf genommen. Die durchschnittliche Monatstemperaturen um 13.00 Uhr lagen je nach Stand im August und September zwischen $16,8-23,0^{\circ} \mathrm{C}$ resp $13,8-18,0^{\circ} \mathrm{C}$.

Der tägliche Bienentotenfall wurde auf dem Stand 8 in der Kontrollgruppe bei drei Völkern und in den Gruppen mit und ohne Kampfer bei je fünf Völkern mit Hilfe einer dem Flugloch vorgebauten Bienenleichenfalle gemessen. Während der ganzen Versuchsdauer sowie im folgenden Frühjahr wurde auf dem Stand 8 bei allen Versuchsvölkern in Intervallen von drei Wochen die Anzahl Bienen sowie die offene und gedeckelte Brutfläche geschätzt (Imdorf et al, 1987). Die Entwicklung der Völker der verschiedenen Versuchsgruppen wurde mit der Varianzanalyse überprüft.

In je zwei Schweizer- und Dadantkästen wurde 1992 während einer Behandlung von 6 Wochen wöchentlich zweimal die Stockluftkonzentration von Thymol, Eukalyptol, Menthol und Kampfer gemessen. Die Stockluft wurde durch ein mit Graphit getülltes Adsorptionsröhrchen (Orbo-101, Supelco) in der Mitte des Brutnestes angesogen. Während der ganzen Messperiode wurde die Umgebungstemperatur der VARTafeln gemessen. Die durchschnittliche Tagestemperatur wurde aus 162 Messungen ermittelt.

Nach Abschluss der Behandlung mit 'Apilife VAR' wurde im Oktober auf jedem Stand aus je drei Völkern pro Versuchsgruppe eine Futterprobe gefasst. Pro Volk wurde mit einem Teelöffel auf jeder Brutwabe Wabe beidseitig je ein Löffel Futter entnommen. Im Frühjahr wurde auf jedem Stand aus der Honigernte aller Völker einer Versuchsgruppe je drei Mischproben gezogen.

Die im Adsorptionsröhrchen angereicherten Luftproben wurden in Toluol extrahiert und anschliessend gaschromatographisch analysiert. Die Trennung erfolgt an einer $30 \mathrm{~m}$ DB5 Kapillarsäule (J\&W Nr 123-5031) mit Temperaturprogramm $60-180^{\circ} \mathrm{C}\left(5^{\circ} \mathrm{C} / \mathrm{min}\right)$ und FID Detektion. Die Honig- und Futterproben wurden mittels Festphasenextraktion (Baker SPE, Säulen $\mathrm{Nr}$ 7020-06) extrahiert, mit Ethanol eluiert und gaschromatographisch bestimmt. Das Temperaturprogramm war $2 \mathrm{Min}$ bei $60^{\circ} \mathrm{C}, 2^{\circ} \mathrm{C}$ pro Min bis $100^{\circ} \mathrm{C}$, dann $30^{\circ} \mathrm{C}$ pro Min bis $280^{\circ} \mathrm{C}$ und 30 Min bei $280^{\circ} \mathrm{C}$.

Die Wiederfindungsraten in Luftproben waren wie folgt: Eukalyptol $87 \%$ ( $S D \pm 9, n=24$ ), Men- thol $85 \%$ (SD $\pm 11, n=22$ ), Kampfer $88 \%$ (SD \pm $7, n=29$ ) und Thymol $64 \%$ (SD $\pm 11, n=40$ ). In de Futter- und Honigproben lagen die Nachweisgrenzen für alle 'Apilife VAR' Komponenten bei 0,02 bis $0,04 \mathrm{mg} / \mathrm{kg}$. Die Wiederfindung betrug beim Futter respektive Honig für Thymol $85 \%$ $(S D \pm 6$ ) respektive $85 \%$ (SD \pm 16 ), für Eukalyptol $82 \%(S D \pm 7)$ respektive $89 \%(S D \pm 10)$ für Menthol $95 \%(S D \pm 13$ ) respektive $87 \%(S D \pm 5$ ) und für Kampfer $92 \%(S D \pm 10)$ respektive $91 \%$ $(\mathrm{SD} \pm 11)$.

\section{ERGEBNISSE UND DISKUSSION}

\section{Wirksamkeit von 'Apilife VAR'}

\section{Behandlungsdauer}

Der Behandlungserfolg war auf den Ständen 1 und 2 bei sehr niedriger Milbenpopulation nach 8 Wochen etwas höher als nach 4 Wochen. Der Unterschied ist nicht signifikant (Tabelle I). Die hohe durchschnittliche Wirksamkeit nach 8 Wochen von 96,8 respektive $98,6 \%$ bestätigen die Ergebnisse von Rickli et al (1991). Diese Untersuchung hat aber auch gezeigt, dass bei einem hohen Milbenbefall oder beim Auftreten einer Rückinvasion eine Behandlungszeit von 4 Wochen nicht ausreicht. Deshalb wird im Normalfall eine Behandlungsdauer von 6-8 Wochen notwendig sein.

\section{Dosierung im Schweizerkasten}

Bei der Behandlung mit 2 mal 1/2 Tafel ist im Schweizerkasten mit einem signifikant schlechteren Behandlungserfolg zu rechnen (Tabelle I). Wird nur 1 Tafel anstelle von 2 verwendet, so muss ebenfalls mit einem geringeren Erfolg gerechnet werden. Der Unterschied war aber nicht signifikant. 2 mal 1 Tafel ist somit die Beste der getesteten Dosierungen für den Schweizerkasten. 


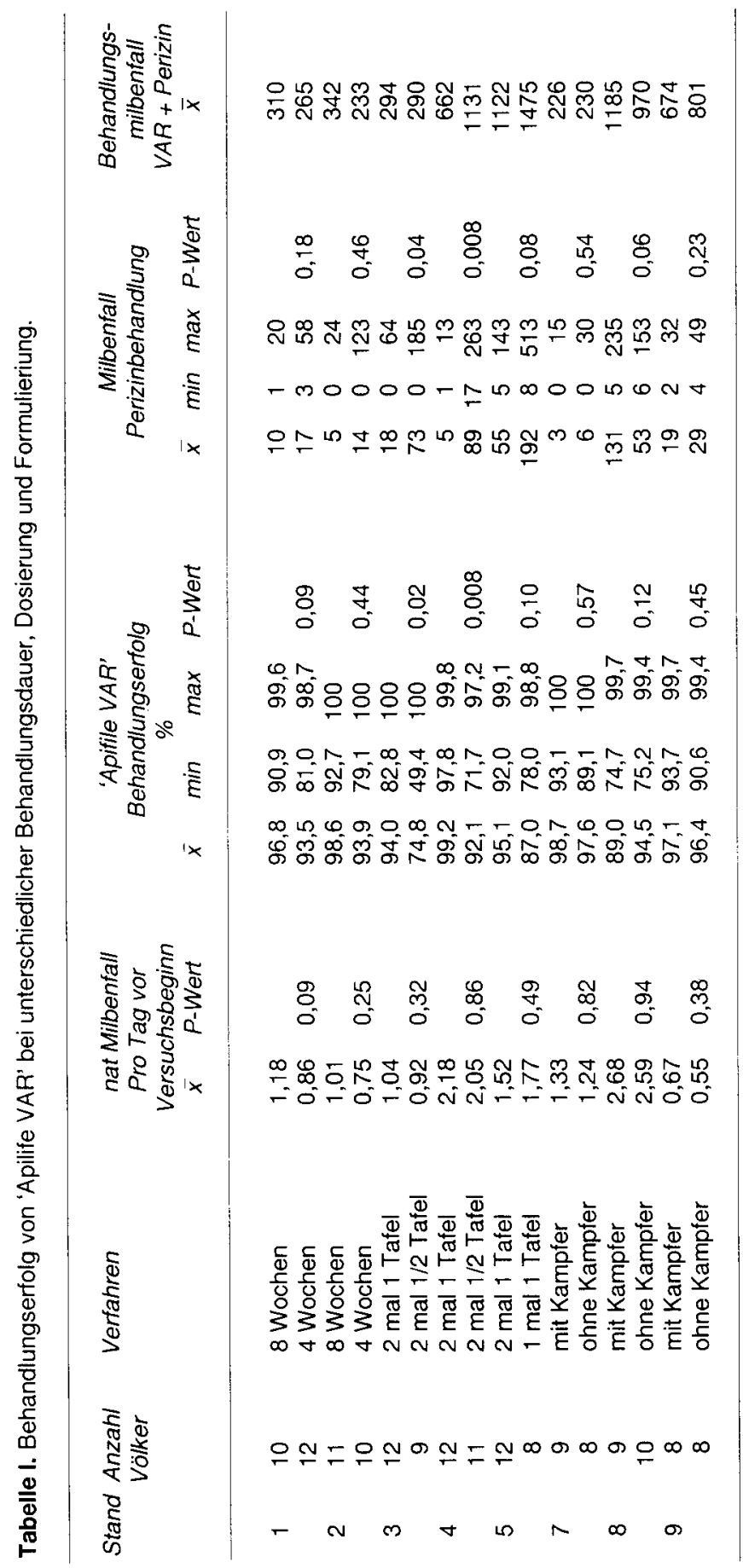




\section{Formulierung}

Zwischen den Behandlungen mit oder ohne Kampfer konnte weder in den Magazinbeuten (Stände 8 und 9) noch im Schweizerkasten (Stand 7) ein signifikanter Unterschied im Behandlungserfolg festgestellt werden (Tabelle I). Dieser Befund erstaunt nicht, ist doch die verwendete Menge von ca 0,7 g Kampfer sehr klein. Unter diesen Bedingungen steigt die Kampferkonzentration in der Stockluft nur selten über $1 \mu \mathrm{g} / \mathrm{l}$. Labortests haben gezeigt, dass für ein voliständiges Abtöten der Varroa-Milben auf gekäfigten Bienen eine Luftkonzentration von ca $50 \mu \mathrm{g} / \mathrm{l}$ während drei Tagen notwendig ist (unveröffentlichte Resultate).

\section{Plazierung der 'Apilife VAR' Tafel im Schweizerkasten}

Die Tafeln aus Vermiculit werden oft von den Bienen mehr oder weniger abgetragen. Dadurch wird die Verdunstungsfläche kleiner. Durch das Einschieben eines bienendichten Gitters zwischen Brutwaben und Tafel konnte der Abbau verhindert werden. Dadurch wurde der Behandlungserfolg aber nicht signifikant verbessert (Tabelle II). Im Gegenteil, auf Stand 5 wurde bei einem Volk das Gitter von den Bienen stark verpropolisiert und der Behandlungserfolg nahm stark ab. Durch das Gitter wird aber in der näheren Umgebung der Tafel die Durchmischung von Wachs und Vermiculit verhindert.

\section{Plazierung der 'Apilife VAR' Tafel im Dadant- oder Ritterkasten}

In diesen Magazinbeuten wurde die $\mathrm{Be}$ handlung von oben (direkt auf den Brutwaben) und von unten (im $4,5 \mathrm{~cm}$ hohen Hochboden) verglichen. Bei der Behandlung von unten war der Behandlungserfolg im Dadant- und Ritterkasten mit 51 respek- tive $71 \%$ klar ungenügend (Tabelle II). Diese Resultate decken sich mit den Ergebnissen von Mutinelli et al (persönliche Mitteilung). Vermutlich war die Stockluftkonzentration der VAR-Komponenten im Brutnestbereich für eine gute Wirkung zu niedrig. Im Ritterkasten war bei der Anwendung von oben der durchschnittliche Behandlungserfolg bei der Gruppe mit Kampfer $97,1 \%$ und bei derjenigen ohne Kampler $96,4 \%$. Im Dadantkasten hingegen lagen die vergleichbaren Werte bei $89,0 \%$ respektive $94,5 \%$ und müssen als leicht ungenügend taxiert werden.

\section{Schätzen der Restmilben nach der Behandlung mit 'Apilife VAR'}

$\mathrm{Zu}$ diesem Zweck wurde der tägliche Milbenfall von 183 Versuchsvölkern während zwei Wochen nach Ende der Anwendung von 'Apilife VAR' mit dem Milbenfall bei der Perizinbehandlung verglichen (Abb 1). Es zeigte sich eine starke Abhängigkeit zwischen den beiden Parametern $(r=0,867)$. Bei einer gleichartigen Untersuchung nach der Anwendung der Ameisensäure konnte ebenfalls eine starke Abhängigkeit ( $r=$ 0,85 ) festgestellt werden (Imdorf und Kilchenmann, 1990). Auch Moosbeckhofer (1991) verglich den täglichen Milbenfall im Herbst von 25 Völkern mit demjenigen der Wintermilbenpopulation und erhielt eine Korrelation von 0,84. Das Messen des natürlichen Milbenfalls während zwei Wochen nach der Behandlung ist somit ein zuverlässiges Instrument, um die Restmilbenzahl und den Behandlungserfolg von 'Apilife VAR' ohne Einsatz von Akariziden zu überprüfen.

Fäll in den 2 Wochen nach der Behandlung mit 'Apilife VAR' durchschnittlich 1 Milbe pro Tag, so entspricht dies insgesamt ca 40 Milben bei der Perizinbehandlung. Von den 121 Völkern mit weniger als 1 Milbe pro Tag wiesen nur drei Völker bei 


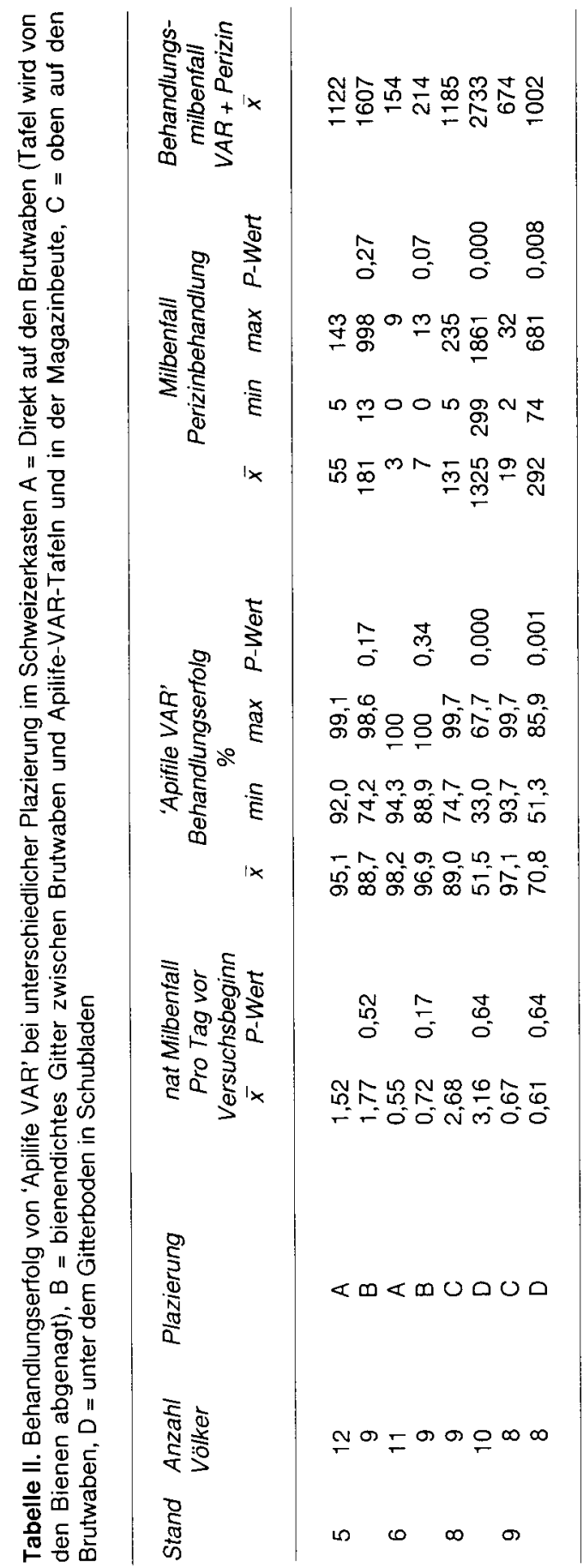




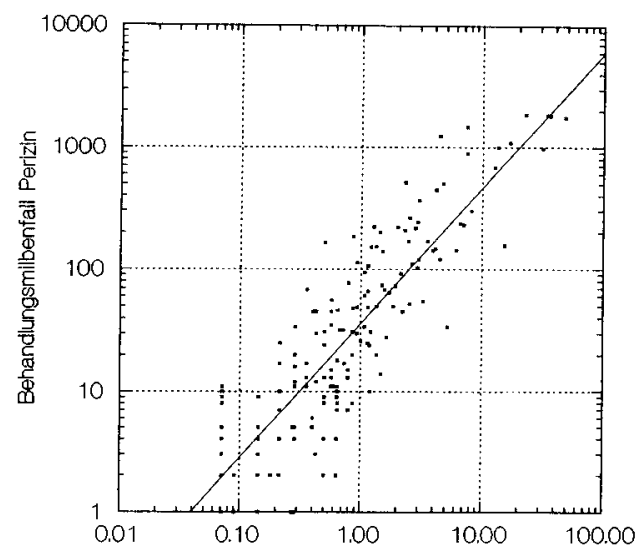

Milbenfall pro Tag - 2 Wochen nach "Apillfe VAP"

Abb 1. Abhängigkeit zwischen dem durchschnittlichen Milbenfall pro Tag während den 2 Wochen nach der Behandlung mit 'Apilife VAR' und dem Milbenfall der Perizinbehandlung resp der Wintermilbenpopulation ( $r=0,867, n=183$ ).

der Perizinbehandlung etwas mehr als 100 Milben auf. Daraus können für die Praxis folgende Schlussfolgerungen gezogen werden: Liegt der Milbenfall während den ersten zwei Wochen nach der Behandlung unter 1 Varroa pro Tag, so ist die Restpopulation noch kleiner als 100 Varroa. Unter diesen Bedingungen erübrigen sich weitere Bekämpfungsmassnahmen.

\section{Bienentoxizität und Volksentwicklung}

Auf dem Stand 8 wurden während der Behandlung mit 'Apilife VAR' bei je fünf Versuchsvölkern der Gruppe mit Kampfer im Durchschnitt pro Tag 5,6 (SD $\pm 4,4)$ und in der Gruppe ohne Kampfer 4,4 (SD $\pm 2,0$ ) tote Bienen gezählt. Bei den drei unbehandelten Kontrollvölkern fielen 2,9 (SD \pm $1,12)$ tote Bienen pro Tag. Zwischen den
Gruppen konnte kein signifikanter Unterschied festgestellt werden.

In diesen Versuchsgruppen wurde die Volksstärke zu den Zeitpunkten der Einund Auswinterung sowie Ende Mai, nach der Frühjahrsentwicklung, verglichen. Dabei konnte kein signifikanter Unterschied in der Volksentwicklung zwischen den mit 'Apilife VAR' behandelten und den unbehandelten Völkern festgestellt werden.

\section{Verlauf der Stockluftkonzentration von 'Apilife VAR'}

Während der sechswöchigen Behandlung mit 'Apilife VAR' wurde im August und September 1992 in 2 Schweizer- und 2 Dadantkästen in der Mitte des Brutnestes die Stockluftkonzentration der 4 Komponenten von 'Apilife VAR' ermittelt. Thymol erreichte in den 4 Völkern Werte zwischen 1,1 und $21,3 \mu \mathrm{g} / \mathrm{l}$ Stockluft. Für Eukalyptol, Menthol und Kampfer lagen die Werte viel tiefer und schwankten zwischen $\leq 0,02$ und 2,4 $\mu \mathrm{g} / \mathrm{l}$ Luft. Wir vermuten daher, dass neben Kampfer auch Eukalyptol und Menthol in dieser Formulierung keine akarizide Wirkung haben. Allerdings wurden synergistische Effekte von Eukalyptol und Menthol nicht getestet. Chiesa (1991) erreichte allein mit Thymol einen Behandlungserfolg, der jenem von 'Apilife VAR' ähnlich ist.

Während der sechswöchigen Behandlung war die durchschnittliche Thymolkonzentration in der Stockluft der beiden Schweizerkastenvölker relativ ausgeglichen und schwankte zwischen 3,6 und 7,5 $\mu \mathrm{g} / \mathrm{l}$ (Abb 2). Im Dadantkasten hingegen waren grosse Schwankungen zu verzeichnen. Sie lagen zwischen 2,7 und 19,0 $\mu \mathrm{g} / \mathrm{l}$. Obwohl die durchschnittlichen täglichen Umgebungstemperaturen der Tafeln während den ersten 2,5 Wochen nur geringe 
Unterschiede aufwiesen (Abb 2), lag die Thymolkonzentration zu Behandlungsbeginn in den Dadantkästen 3 mal höher als in den Schweizerkästen. Gegen Ende der Behandlungszeit der ersten und der zweiten Tafel nahmen die Konzentrationen in den Dadantkästen stark ab. Von Volk zu Volk traten grosse Unterschiede in der Stockluftkonzentration auf. In den Dadantvölkern konnte zu Beginn der Behandlung, als die Stockluft einen Thymolgehalt von beinahe $20 \mu \mathrm{g} / \mathrm{l}$ erreichte, ein starker Brutund Bienenrückgang beobachtet werden. Während dieser Zeit herrschten Aussentemperaturen von über $30^{\circ} \mathrm{C}$ und die Kästen waren den ganzen Tag der direkten Sonneneinstrahlung ausgesetzt. Solch hohe Thymolkonzentrationen sind durch geringere Dosierungen und kleinere Verdunstungsflächen zu vermeiden.

\section{Rückstände in Futter und Honig}

In allen Futterproben wurden nur Thymolrückstände gefunden. Bei Behandlungen mit signifikant schlechterem Behandlungserfolg, wie nach 2 mal 1/2 Tafel und der Behandlung von unten in den Magazinbeuten, wurden signifikant tiefere Rückstände im Futter gemessen (Tabelle III). Im Schweizerkasten wurde nach der Behandlung von 8 Wochen durchschnittlich $2,6 \mathrm{mg} / \mathrm{kg}$ (SD $\pm 2,0, n=30$ ) Thymol im Futter gemessen. Der Höchstwert betrug $9,2 \mathrm{mg} / \mathrm{kg}$. In Magazinbeuten lag der Durchschnittswert nur bei $2,0 \mathrm{mg} / \mathrm{kg}$ (SD \pm $0,98, n=12$ ) und der Höchstwert bei $3,2 \mathrm{mg} / \mathrm{kg}$.

Im Honig resultierten bedeutend niedrigere Werte. Die unterschiedlich hohen Thymolrückstände lassen sich nicht auf die Behandlungsart zurückführen (Tabelle III). Es scheinen hier eher standbezogene Ursachen dafür verantwortlich zu sein. Es wird vermutet, dass auf den Ständen mit hohen Thymolrückständen im Honig, kontaminierte Futterreste im Frühling in den Honigraum umgetragen wurden. Die durchschnittliche im Honig gefundene Thymolmenge lag im Schweizerkasten bei der Dosierung mit zwei Tafeln und einer Be-

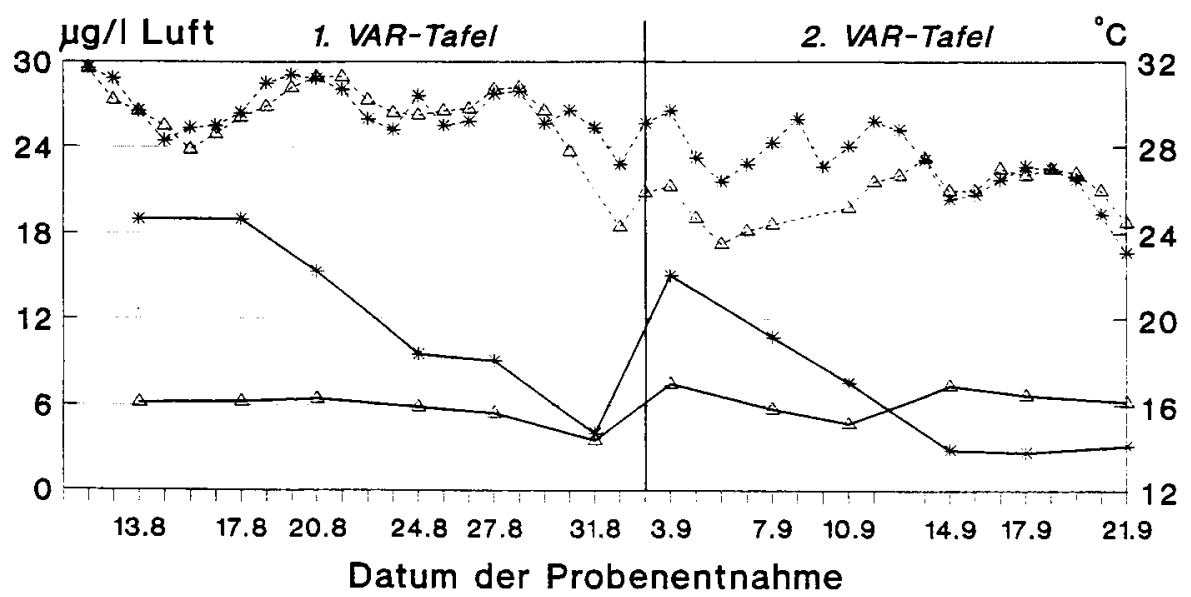

Abb 2. Verlauf der Stockluftkonzentration von Thymol und der durchschnittlichen Tagestemperatur während der sechswöchigen Behandlung mit 'Apilife VAR' in Schweizer- und Dadantkasten (Mittelwerte, $n=2)$. $-\Delta-$ Schweizerkasten; $-x-$ Dadantkasten; -- $\Delta$-- Umgebungstemperatur der VARTafeln im Schweizerk; --x-- Umgebungstemperatur der VAR-Tafeln im Dadantk. 
Tabelle III. Thymolrückstände in Futter und Honig nach der Behandlung mit 'Apilife VAR'.

\begin{tabular}{|c|c|c|c|c|c|c|c|c|}
\hline \multirow[t]{2}{*}{ Verfahren } & & \multirow[b]{2}{*}{$n$} & \multicolumn{3}{|c|}{ Futter } & \multicolumn{3}{|c|}{ Honig } \\
\hline & & & $\begin{array}{c}\bar{x} \\
m g / k g\end{array}$ & $\begin{array}{l}S D \\
\pm\end{array}$ & P-Wert & $\begin{array}{c}\bar{x} \\
m g / k g\end{array}$ & $\begin{array}{l}S D \\
\pm\end{array}$ & $P$-Wert \\
\hline \multirow[t]{2}{*}{ Behandlungsdauer } & 2 mal 4 Wochen & 6 & 2,72 & 2,51 & & 0,10 & 0,09 & \\
\hline & $2 \mathrm{mal} 2$ Wochen & 6 & 0,94 & 0,63 & 0,15 & 0,13 & 0,06 & 0,49 \\
\hline \multirow[t]{3}{*}{ Dosierung } & 2 mal 1 Tafel & 6 & 2,48 & 1,59 & & 0,22 & 0,06 & \\
\hline & $2 \mathrm{mal} 1 / 2$ Tafel & 6 & 0,58 & 0,30 & 0,03 & 0,16 & 0,06 & 0,10 \\
\hline & $1 \mathrm{mal} 1$ Tafel & 3 & 0,83 & 0,10 & & 0,04 & 0,01 & \\
\hline \multirow[t]{2}{*}{ Formulierung } & mit Kampfer & 9 & 2,82 & 2,52 & & 0,09 & 0,09 & \\
\hline & ohne Kampfer & 9 & 2,20 & 1,33 & 0,53 & 0,02 & 0,02 & 0,06 \\
\hline \multirow[t]{2}{*}{ Plazierung $\mathrm{CH}$} & mit Gitter & 6 & 1,78 & 0,84 & & 0,05 & 0,02 & \\
\hline & ohne Gitter & 6 & 2,60 & 1,37 & 0,25 & 0,05 & 0,01 & 0,80 \\
\hline \multirow{2}{*}{ Plazierung Magazin } & oben & 6 & 1,77 & 0,74 & & 0,03 & 0,02 & \\
\hline & unten & 6 & 0,16 & 0,21 & 0,002 & $\leq 0,02$ & & \\
\hline
\end{tabular}

handlungsdauer von 8 Wochen bei $0,1 \mathrm{mg} /$ $\mathrm{kg}(\mathrm{SD} \pm 0,09, n=30)$. Der höchste Wert betrug $0,33 \mathrm{mg} / \mathrm{kg}$. In den Magazinbeuten waren die vergleichbaren Werte mit 0,03 (SD $\pm 0,01, n=12$ ) und 0,05 bedeutend tiefer.

Die hier gefundenen Thymol Rückstände dürften human-toxikologisch nicht von Bedeutung sein. Thymol, Eukalyptol, Menthol und Kampfer werden in der Lebensmittelindustrie und Kosmetik verwendet. Sie sind mit dem 'GRAS' Status (Generally Recognized As Safe) versehen, dh Konzentrationen bis zu $50 \mathrm{mg} / \mathrm{kg}$ werden als unbedenklich eingestuft.

Im Bezug auf die Rückstände im Wachs liegen nur wenige Untersuchungen vor. Lodesani et al (1990) fand nach einer Thymolstaubbehandlung, welche im Oktober und November durchgeführt wurde, Anfang März durchschnittlich $45 \mathrm{mg} / \mathrm{kg}$ und $\mathrm{im}$ Juli noch $0,15 \mathrm{mg} / \mathrm{kg}$. Es ist somit anzunehmen, dass im Wachs längerfristig keine grössere Anreicherung dieser Substanz stattfindet.

\section{SCHLUSSFOLGERUNGEN}

'Apilife VAR' weist unter optimalen Bedingungen im Schweizer- und Ritterkasten (2 mal 1 Tafel und 8 Wochen Behandlungsdauer) eine sehr gute akarizide Wirkung auf. Weitere Versuche sind notwendig um den Behandlungserfolg im Dadantkasten und in anderen Magazinbeuten zu verbessern. Die Nebenwirkungen beschränken sich bei richtiger Dosierung auf Futterumtrag im Nahbereich der Tafeln und eine verlangsamte Futterabnahme bei einzelnen Völkern. Es ist daher zu empfehlen, einen grossen Teil der Auffütterung vor Behandlungsbeginn durchzuführen. Die Anwendung ist sehr einfach und mit wenig Arbeitsaufwand verbunden. Die Wirkung dürfte in erster Linie abhängig sein von der Thymolkonzentration in der Stockluft. Diese wiederum wird von verschiedenen Faktoren wie Temperatur, Kastenvolumen und Kastensystem (Warmbau oder Kaltbau), Volksstärke etc beeinflusst. Es ist daher dringend notwendig, den Behand- 
lungserfolg mit Hilfe des Milbenfalls während den ersten zwei Wochen nach Behandlungsende zu überprüfen, damit kann eine zu hohe Restmilbenpopulation ausgeschlossen werden kann. Ob 'Apilife VAR' der Praxis zur Anwendung empfohlen werden kann, hängt von den Ergebnissen weiterer Rückstandsuntersuchungen ab. Dazu braucht es Geschmacksprüfungen mit Honig, um die Wahrnehmungsgrenze von Thymol festlegen zu können. Im weiteren ist eine Verbesserung der Anwendung in bestimmten Magazinbeuten anzustreben.

\section{DANKSAGUNG}

Den folgenden Imkern/in möchten wir für die Durchführung der Versuche auf ihren Bienenständen herzlich danken: B Bachofen, Annemarie Burren, A Feuz, G Giroud, $M$ Keller, $F$ Lehmann, W Lüthi, H Oppliger, J Peyer, A Sciboz und J Wespi.

\section{Summary - Optimization of the use of} 'Apilife VAR' to combat Varroa jacobsoni Oud in honey bee colonies. 'Apilife VAR' has been developed to combat Varroa jacobsoni in bee colonies. It consists of a Vermiculite plate which is loaded with about $20 \mathrm{~g}$ of a mixture of thymol $(76 \%)$, eucalyptol (16.4\%), menthol (3.8\%) and camphor $(3.8 \%)$. The purpose of these tests was to examine the efficiency of the product in the following conditions: a) duration of the treatment: 4 or 8 weeks; b) dosage in Swiss hives: twice 1 plate, twice $1 / 2$ plate or once 1 plate; c) formulation: with or without camphor; d) disposition of the plates in Swiss hives, directly on the brood combs or on a fine-meshed grid positioned on the combs; e) disposition of the plates in Dadant and Ritter hives: directly on the combs (above) or under the bottom grid (below). Problems related with bee toxicity, concentration of the components of the product in the air of the hive and residues in the bee food and honey have also been examined. In the Swiss hive the average efficiency of 'Apilife VAR' was $97.7 \%$ after 8 weeks of treatment and $93.7 \%$ after 4 weeks of treatment. The difference was not significant. Twice 1 plate was the best dosage in the Swiss hive. The difference of formulation (with or without camphor) did not influence the results of the treatment (table I). The fine-meshed grid between the brood comb and the plate did not change substantially the efficiency of the product as long as the bees did not cover the grid with propolis. In the Dadant and Ritter hives the treatment from below was not efficient enough (table II). The treatment from above had an efficiency of $97.1 \%$ in the Ritter hives and of $89 \%$ in the Dadant hives. A method for the estimation of the number of varroas remaining in the colony after application of 'Apilife VAR' is presented. It is based on the correlation $(r=0.867)$ between the number of dead varroas found on the hive bottom during the first 2 weeks after the 'Apilife VAR' treatment and the number of dead varroas after the subsequent Perizin treatment (fig 1). If less than 1 varroa falls per day, the remaining varroa population will be lower than 100 . If this condition is filled no further measures must be taken. 'Apilife VAR' did not influence the mortality of the bees and the development of the colonies. The thymol concentrations of the air of 4 bee colonies varied from 1.1 to $21.3 \mu \mathrm{g} / \mathrm{l}$. Whereas the values were equilibrated in the Swiss hives, they fluctuated considerably in the Dadant hives (fig 2). The eucalyptol, menthol and camphor concentrations were much lower. They varied between $\leq 0.02$ and $2.4 \mu \mathrm{g} / \mathrm{l}$ of air. These concentrations have probably no varroacidal effect. Only thymol residues were detected in the bee food and honey (table III). After 8 weeks of treatment the mean residue values were $2.6 \mathrm{mg} / \mathrm{kg}(\mathrm{SD} \pm 2.0, n=30)$ in the Swiss hives and $2.0 \mathrm{mg} / \mathrm{kg}(\mathrm{SD} \pm 0.98, n=12)$ in 
the Dadant and Ritter hives. The maximum value was $9.2 \mathrm{mg} / \mathrm{kg}$. 8 months later the respective values in the spring honey harvest were $0.1 \mathrm{mg} / \mathrm{kg}(\mathrm{SD} \pm 0.09, n=30)$ and $0.03 \mathrm{mg} / \mathrm{kg}(\mathrm{SD} \pm 0.01, n=12)$, with a maximum value of $0.33 \mathrm{mg} / \mathrm{kg}$. The residues found in honey are innocuous to human health. Thymol is generally recognized as safe ('GRAS' status), ie concentrations up to $50 \mathrm{mg} / \mathrm{kg}$ are considered unobjectionable. Before making a general assessment of 'Apilife VAR' it will be necessary to carry out further residue measurements and to fix the perception threshold for thymol in different types of honey.

Varroa jacobsoni / chemical control / essential oil / treatment efficiency / residue

\section{Résumé - Optimisation de l'emploi d'Apilife VAR pour la lutte contre Varroa jacobsoni Oud dans les colonies} d'abeilles. Apilife VAR a été mis au point pour lutter contre Varroa jacobsoni dans des colonies d'abeilles. Ce produit consiste en une plaque de Vermiculite qu'on imprègne d'environ $20 \mathrm{~g}$ du mélange suivant: thymol $(76 \%)$, eucalyptol $(16,4 \%)$, menthol $(3,8 \%)$ et camphre $(3,8 \%)$. Cet essai était destiné à examiner l'efficacité du produit dans les conditions suivantes : a) durée du traitement : 4 ou 8 sem; b) dosage dans les ruches suisses : 2 fois 1 plaque, 2 fois $1 / 2$ plaque ou 1 fois 1 plaque; c) formulation : avec ou sans camphre; d) disposition de la plaque dans la ruche suisse : directement sur les cadres de couvain ou sur une grille à mailles serrées ne laissant pas passer les abeilles, posée sur les cadres de couvain; e) disposition de la plaque dans les ruches Dadant et Ritter : directement sur les cadres de couvain (en haut) ou sous la grille qui recouvre le plancher (en bas). Nous avons également exa- miné des questions relatives à la toxicité du produit pour les abeilles, à la concentration de ses composants dans l'air des ruches et aux résidus dans la nourriture des abeilles et le miel.

L'efficacité moyenne dans les ruches suisses était de $97,7 \%$ après 8 sem et de $93,7 \%$ après 4 sem. La différence n'était pas significative. Le dosage optimal dans les ruches suisses était de 2 fois 1 plaque. La différence de formulation d'Apilife VAR, avec ou sans camphre, n'a pas influencé l'efficacité des traitements (tableau I). La grille à mailles serrées posée entre les cadres de couvain et la plaque n'a pas modifié l'efficacité du produit tant que les abeilles ne recouvraient pas la grille de propolis. L'efficacité du traitement par le bas était insuffisante dans les ruches Dadant et Ritter (tableau II). Le traitement par le haut avait une efficacitè moyenne de $97,1 \%$ dans les ruches Ritter et de $89 \%$ dans les ruches Dadant. Une méthode est présentée, qui permet d'estimer les varroas restant dans les colonies après le traitement Apilife VAR. Elle s'appuie sur la corrélation $(r=0,867)$ entre la chute de varroas pendant les 2 premières sem suivant le traitement VAR et la chute de varroas après le traitement au Périzin (fig 1). Si la chute de varroas est inférieure à 1 varroa par jour, la population restante compte moins de 100 varroas. Dans ce cas, on peut renoncer à d'autres mesures de lutte. Le traitement Apilife VAR n'a pas influencé la mortalité des abeilles et le développement des colonies. La concentration en thymol de l'air des ruchers, mesurée dans 4 colonies, variait entre 1,1 et $21,3 \mu \mathrm{g} / \mathrm{l}$. Dans les ruches suisses, elle était équilibrée. Dans les ruches Dadant, elle fluctuait fortement (fig 2). Les taux d'eucalyptol, de menthol et de camphre étaient beaucoup plus bas. Ils variaient de $\leq 0,02$ à $2,4 \mu \mathrm{g} / \mathrm{l}$ d'air. Ces concentrations n'ont très probablement pas d'effet varroacide. Seuls des résidus de thymol ont été 
détectés dans la nourriture des abeilles et dans le miel (tableau III). Après $8 \mathrm{sem}$ de traitement, on a enregistré des taux de résidus moyens de $2,6 \mathrm{mg} / \mathrm{kg}$ ( $\mathrm{sd} \pm 2,0 ; n=$ 30) dans la nourriture des ruches suisses et de $2,0 \mathrm{mg} / \mathrm{kg}$ ( $\mathrm{sd} \pm 0,98 ; n=12$ ) dans celle des ruches Dadant et Ritter. La valeur maximum était de $9,2 \mathrm{mg} / \mathrm{kg}$; 8 mois plus tard, les taux de résidus étaient respectivement de $0,1 \mathrm{mg} / \mathrm{kg}$ ( $\mathrm{sd} \pm 0,09$; $n=30$ ) et $0,03 \mathrm{mg} / \mathrm{kg}$ ( $\mathrm{sd} \pm 0,01 ; n=12$ ) dans la récolte de miel du printemps, avec une valeur maximum de $0,33 \mathrm{mg} / \mathrm{kg}$. Les résidus détectés dans le miel sont inoffensifs pour la santé humaine. Le thymol possède le statut GRAS (generally recognized as sage), c'est-à-dire que les concentrations allant jusqu'à $50 \mathrm{mg} / \mathrm{kg}$ sont considérées comme inoffensives. Avant de procéder à une appréciation générale d'Apilife VAR, il faut attendre les résultats d'autres mesures des résidus et déterminer le seuil de perception du thymol dans différentes sortes de miel.

\section{Varroa jacobsoni / lutte chimique / huile essentielle / efficacité traitement / résidu}

\section{LITERATURLISTE}

Chiesa F (1991) Effective control of varroatosis using powdered thymol. Apidologie 22, 135145

Colin M (1990) Essential oils of Labiatae for controlling honey bee varrosis. $J$ Appl Entomol 110, 19-25

Hoppe H (1990) Vergleichende Untersuchungen zur biotechnischen Bekämfung der Varroatose. Dissertation, Justus-LiebigUniversität, Giessen und Tierhygienisches Institut Freiburg, Deutschland

Hoppe H, Ritter W (1989) Erste Ergebnisse zur Bekämpfung der Varroatose mit einem ther- mischen Umluftverfahren in Kombination mit Wintergrünöl. Die Biene 7, 390-393

Imdorf A, Bühlmann G, Gerig L, Kilchenmann V, Wille $H$ (1987) Überprüfung der Schätzmethode zur Ermittlung der Brutfläche und Anzahl Arbeiterinnen in freifliegenden Bienenvölkern. Apidologie 18, 137-146

Imdorf A, Kilchenmann V (1990) Natürlicher Milbenfall im Oktober - eine zuverlässige Schätzmethode der Wintermilben. Schweiz Bienentztg 113, 505-506

Kraus B (1990) Untersuchungen zur olfaktorischen Orientierung von Varroa jabcobsoni und deren Störung durch ätherische Öle. Dissertation, JW Goethe-Universität Frankfurt, Institut für Bienenkunde Oberursel, Deutschland

Liebig G, Gerlich P, Herter H (1992) Bericht der Landesanstalt für Bienenkunde in Hohenheim für das Jahr 1991. Versuchswesen: 'Apilife VAR'. Bienenpflege 3, 80-81

Lodesani M, Bergomi S, Pellacani A, Carpana E, Rabatti T (1990) Prove sperimentali per la valutazione dell'efficacia e per la determinazione dei residui di alcuni prodotti impiegati nella lotta alla varroasi. Apicoltura 6, 105-130

Marchetti S, Barbattini R (1984) Comparative effectiveness of treatments used to contol Varroa jacobsoni Oud. Apidologie 15, 363378

Mautz D (1982) Untersuchungen zur Bienengefährlichkeit von Thymol. Apidologie 13, 103104

Moosbeckhofer R (1991) Varroaverluste während der Überwinterung. Bienenvater 112 , 300-303

Rickli M, Imdorf A, Kilchenmann V (1991) Varroa-Bekämpfung mit Komponenten von ätherischen Ölen. Apidologie 22, 417-421

Ritter W, Perschil F, Jehle V, Koch W, Hoevel R (1986) Versuch zur Entwicklung und Prüfung von Perizin, einem systemischen Medikament zur Bekämpfung der Varroatose der Honigbienen. Allg Dtsch Imkerztg 3, 78-82

Van der Steen J (1992) Der Effekt einer Mischung ätherischer Öle auf die Varroainfektion in Bienenvölkern. Apidologie 23, 383-385 